\title{
Incomplete BIM Implementation: Exploring Challenges and the Role of Product Lifecycle Management Functions
}

\author{
Julie Rose Jupp \\ University of Technology, Sydney \\ Julie.Jupp@uts.edu.au
}

\begin{abstract}
Implementing the BIM methodology relies on mastering new digital ways of working. However approaches to BIM often lack a holistic perspective that span the whole building lifecycle. The omission of Product Lifecycle Management functions in the implementation of the BIM methodology can lead to failures in delivering the benefits of BIM to operations and diminish its value to clients with large property portfolios. In an empirical study, the paper presents an investigation into the current situation of BIM using an Australian commercial property development project. It explores implications of partial implementation for the building lifecycle. Case study findings identify deficiencies in the project environment, management shortcomings associated with the specifics of client requirements, and difficulties in the transverse use of BIM and PLM platforms due to human interface problems.
\end{abstract}

Keywords: Building information modeling, product lifecycle management, multidisciplinary collaboration, operations, facilities management.

\section{Introduction}

Building Information Modeling (BIM) is a model-driven approach to designing, constructing, operating and maintaining buildings. The model that forms the core of the BIM approach is a shared and computable 3D model of the building that helps overcome the shortcomings of a 2D paper-based approach. BIM is therefore a methodology that facilitates multidiscipline collaboration and information management between different project stakeholders [1]. Implementing BIM relies on mastering new digital ways of working to streamline workflow and minimise task redundancies across the building lifecycle [2]. However in practice the integration and management of processes, information technologies (IT) and protocols across building lifecycle phases continues to present major challenges to its implementation.

Whilst these challenges are a complex of factors, they may stem from a lack of a holistic approach to BIM and lifecycle management during design and construction. Recent critiques of BIM highlight implementation deficiencies relative to the integration of facilities management (FM) expertise (e.g., the 'closed-loop problem', [3]), and a lack of core Product Lifecycle Management (PLM) functions during project implementation [4]. In this frame, the paper explores barriers to full BIM implementation, the implications of partial applications of the methodology, and 
examines the role PLM functions might play. A case study was conducted to identify the current situation of the use of BIM in Australian commercial property development. Findings identify deficiencies in the project environment, highlighting management shortcomings associated with the specifics of client requirements and constraints and human interface problems in the use of BIM.

The remainder of the paper is divided into four sections. Sect. 2 presents a brief review of related literature. Sect. 3 introduces the research methods and processes. Sect. 4 presents the case study and findings which are structured according to their significance relative to processes, technologies and policies. Sect. 5 concludes the paper, synthesising observations in a discussion on the nature of the challenges identified, and points to potential ways to overcome them.

\section{Background}

In the last five years an increasing focus on the application of BIM throughout the whole building lifecycle has emerged. The literature surveyed here reveals a growing number of studies that consider a range of building lifecycle management issues, where much of this research has sought to bridge the interface between AEC processes and operations; e.g., development of IFCs to include schemas for FM [5] and the introduction of the COBie initiative (Construction Operations Building information exchange), which allows for the exchange of IFC-based FM data [6]. Research has provided a variety of extensions of BIM technologies to support operations [7-12] and requirements management links to BIM models have been developed [13]. New uses of web technology have also been proposed to deliver building information to support FM [14], including the development BIM for FM portals to provide feedback from project stakeholders and to develop business requirements [15]. Recent studies also focus on the value of as-built datasets to FM personnel and clients [16], highlighting difficulties surrounding their creation during construction [17] and demonstrating that there are often no clear strategies for keeping the BIM model "alive" during operations [3]. Even despite a combined BIM and Integrated Project Delivery approach [18], the flow and management of information is still not fully integrated among all stakeholders [19]. This growing body of literature provides much evidence that research efforts to close the loop and develop the BIM methodology for the whole building lifecycle are increasing.

One of the main gaps identified in the literature relates to the lack of research surrounding the role of PLM in the BIM methodology. Whilst proponents of BIM assert that the methodology can be effective in process integration, knowledge transfer and information management (avoiding information losses incurred when transitioning from one team to another), a critical assumption remains that each group has the ability to "add to and reference back to all information they acquire during their period of contribution to the BIM model" [20]. Such aspirations for fully coordinated datasets and seamless information management appear to be far from reality, largely due to a lack of PLM functionality in applications of BIM. Key aspects of PLM for BIM-enabled projects include the management of processes surrounding the creation, release, change and verification of building information. For example, PLM systems typically provide for the following core functions: the management of 
design and process documents and models, construction and control of bill of material (product structure) records, provision of electronic file repositories, inclusion of document and model metadata, identification of model content for compliance and verification, enabling workflow and process management for approving changes, controlling multi-user secured access and data export controls.

Researchers of BIM management have tackled a variety of PLM and configuration management related issues [4]. London et al. [21] identify BIM management requirements that reflect core PLM functions, including data organisation, version management, release management and role/responsibility identification. BIM model servers, such as Jottne's EDM model server, are also beginning to be used in industry to gain PLM benefits, and whilst promising BIM model servers must still be integrated into a PLM environment to address change management functions [4]. Similarly, recent developments in enterprise solutions for building development, such as ArtrA Enterprise, are providing PLM functionality so as to address asset management. In a bid to integrate BIM and PLM, recent work by Reedman [4] proposes an integrated environment using the Integrated Product Model concept. The IPM concept places a "well managed digital product model" at the centre of the design and engineering process; where 'well managed' means that there are "appropriate release and change processes in place". Their approach utilises BIM to apply configuration management concepts to assure accurate documents and models "all of the time". Yet to realise the value of BIM in operations (where small information gains can result in major improvements in cost and productivity), the main building information source must be complete, error free, verified, updated and maintained, which presents significant challenges to a fragmented, low-tech industry; these challenges are explored in detail in the following case study.

\section{Research Setting and Method}

The research focused on a commercial high-rise project in Australia; for anonymity reasons the project shall be referred to as the 'HR Building'. The development was one of the first in Australia to implement BIM in a multidisciplinary collaboration. A case study approach was adopted [22] and data collection involved analysis of project documents (e.g. project plans, contract documents, project correspondence and reports) and semi-structured interviews with members of the project team. 18 participants were interviewed across five companies (see Table 1). Interviews took place between November 1010 and August 2011. Each interview took approximately one hour and recordings were subsequently transcribed and verified.

The semi-structured interview approach meant that a range of topics related to the research problem could be covered. Key interview questions therefore included the following two areas: (1) the drivers of and barriers to the implementation of the BIM methodology relative to the management of processes, technologies, policies and stakeholder interactions; and (2) where problems were highlighted by interviewees, their impacts on operations were explored with a specific focus on process and information management. From the data collected, workflow processes and information management were mapped, and several recurring themes were identified. 
Table 1. Interviewees

\begin{tabular}{lll}
\hline Organisation & Title & \# Interviewed \\
\hline Developer & Project Development Manager & 1 \\
& Building Manager & 1 \\
& Facilities Manager & 1 \\
\hline \multirow{2}{*}{ Architectural Firm } & Design Technology Director & 1 \\
& Design Director & 1 \\
& Project Architect & 1 \\
& Architectural BIM Manager & 1 \\
& Architectural BIM Modeller & 2 \\
\hline \multirow{2}{*}{ Structural Engineers } & Structural Project Director & 1 \\
& Structural BIM Manager & 1 \\
\hline Services Engineers & Services BIM Manager & 1 \\
& Services BIM Modeller & 1 \\
\hline Construction Contractor & Project Manager & 1 \\
& Design Manager & 1 \\
& Construction BIM Manager & 1 \\
& Construction BIM Modeller & 2 \\
\hline
\end{tabular}

\section{$4 \quad$ Case Study Findings}

The implementation of BIM was a client requirement, mandated in the contract. A design and build procurement method was taken. Broadly, BIM was required to support design and engineering, simulation and analysis, documentation, construction and operations. Using examples from interview notes and transcripts, Sect. 4 builds a picture of the challenges encountered on the HR Building project. General findings are reported before presenting an analysis of interview responses categorised into process-, technology- and policy- based themes.

\subsection{General Findings: Workflow and Management}

The project commenced in 2005, and was completed in 2011. This timespan can be divided into six broad phases: 1) Stage 1 Development Application (DA); 2) Design Competition Winners Announced; 3) Stage 2 DA and Tender Documentation.; 4) Preconstruction and completion of design and construction phase services; 5) Demolition and construction commencement; and 6) Practical completion.

The creation of a collaborative federated model for construction and as-built model for FM were required by the brief. BIM management responsibilities were taken on by the architectural firm. The main architectural and structural models were complimented by intelligent information models developed by services design consultants and manufacturers. All discipline models were coordinated into a federated model for a variety of construction analysis purposes including structural, fire services, hydraulics, electrics, mechanical, interiors and manufacturer's models. 
Building datasets were managed via the architectural design firm until construction where the main contractor took over responsibilities. Datasets were managed via creation, release and change processes using a central model management strategy, which was specified by the architectural firm in project planning and continued by the contractor. Verification processes beyond the use of clash detection analysis prior to construction were not continuously executed throughout construction.

The process of ensuring all facets and services of the building were resolved and coordinated ahead of a tight construction schedule presented a variety of management challenges. Critically, neither an open standard for data exchange nor an open format for FM (e.g., COBie) was utilised; consequently critical data was not captured in the as-built dataset, including some equipment lists, product data sheets, warranties, spare parts lists and preventive maintenance schedules. This information was seen by FM personnel as essential to support operations, maintenance and asset management.

On handover, the federated model could be used for FM in two basic ways, namely for creating (1) O\&M manual hyperlinks, and (2) links within 2D representations of 3D component objects to some asset and technical data. Thus, the as-built model was not only incomplete, but not dynamically or intelligently mapped to the computerised maintenance management system (CMMS). The CMMS procured (by the contractor) was an open, standards-based technology which will enable the integration of the asbuilt model on one network providing a single user interface, local or remote accessibility and a breakdown of all building systems, including heating, ventilation, air conditioning, lighting, energy consumption, access control, video surveillance, intrusion, fire and smoke detection, electrical distribution, power quality and monitoring. Thus in contrast to the high levels of integration reached between design and engineering team members, the integration of FM requirements, knowledge and expertise was overall found to be lacking.

\subsection{Process-Based Issues}

Three process-related challenges to BIM implementation were identified: (1) entrenched traditional practices and lack of best practice, (2) asynchronous nature of FM knowledge integration and (3) timeliness training at hand-over.

Entrenched Work Practices and Lack of Best Practice: A lack of appreciation of IT capabilities was apparent in many interviews, and in particular interviews with the main contractor. This was manifest in a level of skepticism voiced by some interviewees concerning the value of BIM; as one interviewee stated: 'Potentially and theoretically $[\mathrm{BIM}]$ is a great idea, but we see some issues. The whole sale speech of BIM is that it [the building design] will not change on site, because theoretically you create a virtual building. However all the clash conditions were not fully resolved by the consultants at documentation stage.' Also highlighted was a hasty close-out stage, which compromised the quality of CMMS system procured: 'An off-the-shelf solution was procured which supports neither the BIM model nor the FM team's requirements for operations and maintenance.' It was felt that the contractor didn't fully consider the requirements of an integrated BIM-FM solution. Other interviewees voiced frustrations at the level of BIM maturity within industry, particularly in 
relation to a lack of best practice and evidence-based research demonstrating the use of IFC schemas for FM such as COBie: 'We've found flaws in the [CMMS] system, such as missing functionalities, poor tracking and reporting mechanisms, and less than ideal integration with BIM datasets,' and 'The datasets are not in a compatible format, so we were unsure whether assets contain the required data for our systems.' Furthermore, whilst interviewees understood lifecycle management requirements, most didn't have a clear understanding of how existing approaches to PLM could support a BIM environment. A sentiment shared by most interviewees was that many PLM technologies and FM data formats, which were relatively new at the time of the project, lacked evidence-based research to support adoption decisions.

Asynchronous Nature of FM Knowledge Integration: It is well known that the timescale differences between design/production and operations create the "closed loop problem', stemming from the asynchronous nature of FM knowledge integration [3]. It was confirmed that whilst BIM provided a way for FM knowledge to permeate across organisational boundaries, IT objects (and associated protocols) can be misinterpreted as they are sent from one context to another. Therefore, despite initial input from FM consultants at the outset of the project, the problem of closing the design-production-operations loop was not successfully addressed; as one interviewee put it: 'We did feel we had FM requirements covered and the specifications of the asbuilt model were detailed at the outset but the ball seems to have been dropped during construction.' This was also reflected in the following FM personnel statements: 'Our FM team didn't come online until just before handover, which meant our ability to influence specifications and data requirements were limited. FM seems to have dropped off what was already a long list of integration requirements'.

Timeliness of Training at Handover: The timeliness of FM information was therefore a common theme. Almost all interviewees believed that more FM expertise would have benefitted the creation and validation of datasets. Interviewees also believed that the continued participation of FM consultants during construction would have ensured higher levels of integration and avoided communication and training deficiencies at handover. Consequently, the FM team was largely unaware of what was contained in the as-built model: 'At hand-over when we were given the as-built model by the contractor, we didn't really know what had been modeled for our purposes. What we discovered was that there wasn't much detailed asset information.' A common frustration voiced by FM personnel concerned the timing of information exchange to begin earlier than point of hand-over so as to implement verification and training processes. Early involvement during planning and design stages as well as ongoing updates was seen as necessary to enable more efficient data sharing. The timing of these processes was seen as crucial to maximise knowledge transfer between incoming FM personnel and outgoing AEC project participants.

\subsection{Technology-Based Issues}

The study revealed three technology-based issues: (1) unknown FM data requirements, (2) inappropriate technologies and reluctance to use open standards for information exchange, and (3) IT skills shortages. 
Unknown FM Data Requirements: Interviewees raised uncertainties concerning FM data requirements and delivery of the as-built model. It was felt that this was not specified adequately. Checklists typically outlined items without specifying the level of detail of asset data required for operations: 'During construction we weren't clear on what the deliverables were in relation to FM and because we weren't clear it was difficult to provide a good brief to suppliers and manufacturers.' The challenge was seen to be a lack of FM knowledge on the type, level and format of asset data. This issue related to process-based challenges identified in Sect. 4.2 and raises the question of who is responsible for obtaining data which is accurate, complete, and in the appropriate format [16]. It also points to two significant issues: (1) the FM team didn't join the project until just before handover, and (2) the FM team's approach derived from existing organisational practices and reliant on the developer's growing centralised approach to FM across their buildings portfolio (see Sect. 4.4).

Inappropriate Technologies or Reluctance to Use Them: To manage buildings through-life appropriate technologies must be utilised. It was found that required management systems, data standards (e.g., IFC) and formats (e.g., COBie) were not always available or known to users. As one interviewee indicated: 'For firms without prior experience in BIM it was a time-intensive and expensive task to integrate different software [...], and some were unable to provide the required information either due to lack of IT or skills.' Project communications were controlled using an electronic document management (EDM) system. However, interviewees felt the lack of open standards in the BIM platform thwarted EDM benefits: 'The data outputs were platform specific, meeting the needs of only some design consultants. Many subcontractors did mind that we weren't using open standards since their testing couldn't be performed using our model outputs.' Open standards were not always seen as compatible, e.g., one interviewee believed their systems provided the greatest assurances in data quality and efficiency: 'We explored IFCs, and a centralised IFC server. It was thought it would require additional resources beyond the project's scope and experiments [with IFC exchange] were unsuccessful.'

IT Skills Shortages: Many interviewee statements reflect a lack of training and skills, and a lack of experience in the application of BIM, as expressed by one interviewee: 'We have experienced construction professionals who know how to program and schedule a building, and our onsite managers understand the FM issues and building use [...] but they can't manipulate a 4D model or lifecycle analysis models because they do not know how to use the software.' This issue combined with the tight build schedule was seen to be prohibitive to IT skills development: 'We saw the benefits of more advanced 4D and 5D modelling but incompatibilities with the skills of the team prevented this.'

\subsection{Policy-Based Issues}

Three key policy- or procedural-based issues were identified: (1) maturity of BIM standards and frameworks, (2) lack of verification and hand-over procedures, and (3) uncertainty in client-side FM strategy. 
Maturity of BIM Standards and Frameworks: Just as there are different levels of maturity in BIM capabilities across the AEC actor network (with many in the supply chain at differing points on the maturity curve), throughout the course of the seven year project, the maturity of BIM standards has rapidly developed and continues to do so. Interviewees noted the parallel development taking place in the drafting of national BIM standards by public organisations and private enterprise. In addition, project participants also noted other initiatives such as the COBie data format (piloted in 2007 and only becoming a standard in 2011 [6]) and benchmarking BIM performance tools, which were largely unknown and untested at the outset of the project in 2005 .

Lack of Verification and Hand-over Procedures: A common issue raised was a lack of procedures for verifying the as-built dataset. One interviewee stressed: 'There were no real verification procedures to check what's installed versus the final model." This was seen to be an industry-wide issue, where interviewees believed data verification to be a delicate problem, as it may lead to competencies being questioned. Lack of verification meant FM personnel did not have reassurance on asset data quality. An interview with the building manager highlighted concerns: 'When auditing the model, it initially looked like the data was all there, but when checking through the model it often wasn't the case.' Performance specification for digital information is a central PLM concern but may be overlooked by AEC stakeholders and unlike performance specification of physical assets, its application to digital assets is not well understood [23]. Furthermore, due to the nature of the two-stage contract, a twopart management approach ensued. The architectural organisation led BIM management protocols until construction. However these protocols during construction and in particular hand-over procedures were not fully implemented or defined: 'The project had detailed information protocols for each stage. [...] During design there were modeling specific checklists and standards to ensure each discipline was generating data as agreed. But you could say its significance petered out as we moved closer to project completion.' This breakdown was reflected in the failure to define data verification and hand-over protocols: 'By the end of construction, a 'throw-it-over the wall' attitude seemed to be emerging [...] and was compounded by the FM team not coming online until close to project completion.'

Uncertainties in Client-Side Lifecycle Management Strategies: A key problem highlighted by the FM team was the client/developer's existing FM practices and their centralised approach to FM across the buildings portfolio. Any new FM strategy to be developed to incorporate the BIM model was therefore dependent on other organisational 'decision-making' factors surrounding e.g. current FM systems and methods that underpin the wider building portfolio, and existing service contractors and their own work practices. Further, it was felt that the developer needed a roadmap for a strategic FM solution for future BIM-enabled projects and would require a procedure for managing the early engagement and involvement of FM personnel in project planning. It therefore appeared that there was a lack of client-side procedures to capture digital O\&M information to feed forward into future BIM-enabled developments as there was no close-out report documenting lessons learned. 


\section{$5 \quad$ Discussion and Conclusion}

Given the fact that BIM is an interdependent network of processes, technologies and policies, which constitutes a 'methodology to manage building design and project data in digital format throughout the building's lifecycle' [24], the issues identified in Sect. 4 are linked. Connections between the challenges that lead to partial BIM implementation were mapped as shown in Table $2 \mathrm{a}$, where direct $(\bullet)$ and indirect ( $(0)$ connections and dependencies were identified.

Table 2. (a) Interdependencies between incomplete BIM implementation issues, and (b) Mapping challenges to full BIM implementation with PLM functions

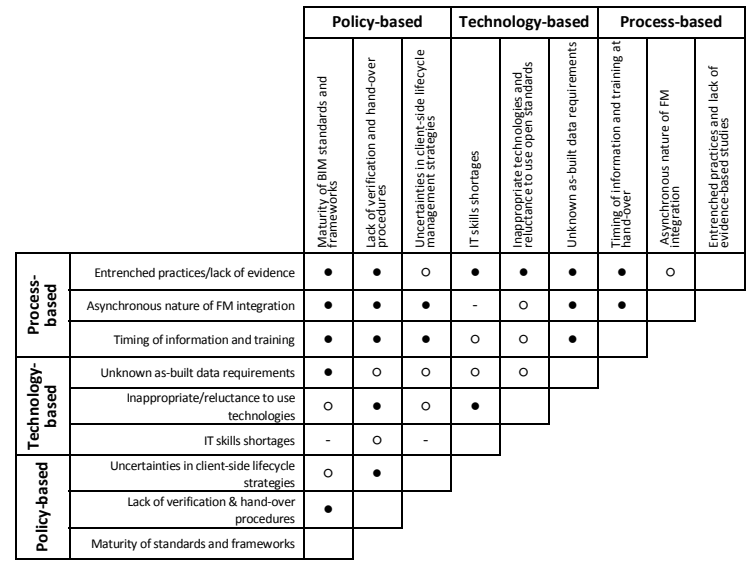

(a)

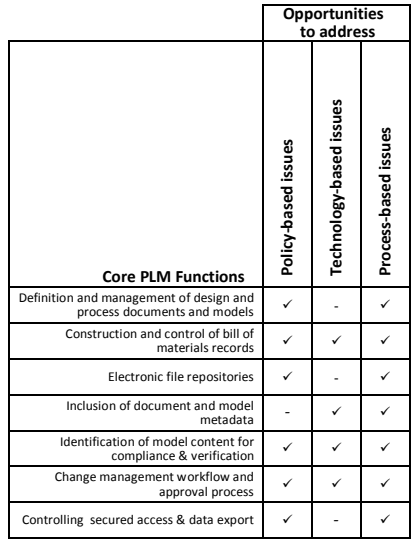

(b)

The mapping reveals the interfaces that are instantiated by each connection: between individuals, teams and organisations; between required processes, information technologies and protocols; and interfaces between lifecycle stages. The complexity of managing these interfaces is vast, and the need for greater PLM functionality is highlighted. Recent approaches to integrating BIM and PLM functions can only account for some of these interfaces. Although PLM allows one to manage and exploit data whilst at the same time defining the product and processes for data development, applying PLM functions in BIM-enabled AEC projects remains problematic. However the significance of PLM is revealed in Table 2b, which maps the potential for core PLM functions to address the challenges encountered in implementing BIM (tagged as ' $\checkmark$ '). Yet, from the case study it is apparent that the complexity and diversity of client requirements, as well as the human interface problems identified, that the transverse utilisation of a BIM-PLM solution across participating organisations would bring new implementation challenges. This is because, whilst most functional requirements of PLM applications are generic, many of the challenges faced by integrators and managers of BIM in the AEC industry arise from the specifics of unique client requirements and collaborating organisations. 
This is a single study of a commercial high-rise development that implemented BIM during the relatively early years of its adoption in industry. Whilst many of the challenges identified stem from the specifics of client and organisational contexts, others are rooted in entrenched practices or result from complexity and uncertainty in collaborative project environments. The extent to which findings can be generalised and transferred to other contexts must therefore be clarified by future research.

\section{References}

1. Succar, B.: Building information modelling framework: A research and delivery foundation for industry stakeholders. Automation in Construction 18, 357-375 (2009)

2. Eastman, C., Teicholz, P., Sacks, R., Liston, K.: BIM Handbook: A Guide to Building Information Modeling, for Owners, Managers, Designers, Engineers, and Contractors. John Wiley \& Sons, Hoboken (2008)

3. Anderson, A., Marsters, A., Sturts Dossick, C., Neff, G.: Construction to Operations Exchange: Challenges of Implementation COBie and BIM in a Large Owner Organization. Construction Research Congress, 15 p. Purdue University, IN (2012)

4. Reefman, R., van Nederveen, S.: A Controlled Integral Product Model (IPM®) in Building and Construction. In: Proceedings of CIB W78-W102 2011: Intl Conf., France (2012)

5. Eastman, C.M.: Building product models: Computer environments supporting design and construction. CRC Press, Boca Raton (1999)

6. East, W.E.: Construction Operation Building Information Exchange. USACE ERDC (2007)

7. Bonandrini, S., Cruz, C., Nicolle, C.: Building lifecycle management. In: Bouras, A., Gurumoorthy, B., Sudarsan, R. (eds.) Proceedings of the International Conference on Product Lifecycle Management, PLM 2005, Lyon, pp. 461-471 (2005)

8. Drogemuller, R., Schevers, H.: Sydney Opera House - FM Exemplar Project: Building Information Modelling for FM at Sydney Opera House (2006)

9. Vanlande, R., Nicolle, C., Cruz, C.: IFC and building lifecycle management. Automation in Construction 18(1), 70-78 (2008)

10. Arayici, Y., Onyenobi, T., Egbu, C.: Building information modelling (BIM) for facilities management (FM): The MediaCity case study approach. International Journal of 3-D Information Modeling (IJ3DIM) 1(1), 55-73 (2012)

11. Ebinger, M., Madritsch, T.: A Classification Framework for Facilities and Real Estate Management: The Built Environment Management Model (BEM2). Facilities 30(5/6), 1 (2012)

12. Shen, W., Hao, Q., Xue, Y.: A loosely coupled system integration approach for decision support in facility management and maintenance. Automation in Construction 25, 41-48 (2012)

13. Kiviniemi, A.: Requirements management interface to building product models (CIFE Technical Report No. 161, PhD thesis), Stanford University, Stanford, CA (2005)

14. Song, Y., Clayton, M.J., Johnson, R.E.: Anticipating reuse: Documenting buildings for operations using web technology. Automation in Construction 11(2), 185-197 (2002)

15. Jordani, D.A.: BIM and FM: The Portal to Lifecycle Facility Management. National Institute of Building Sciences and buildingSMART Alliance, Washington (2010) 
16. Whyte, J., Lindkvist, C., Ibrahim, N.H.: From Projects into Operations: Lessons for Data Handover. Uni. of Reading, DIRC. Working Paper (2011),

http://www.reading.ac.uk/designinnovation/for-industry/ di-for-industry-workingpapers.aspx (accessed February 15, 2013)

17. Xuesong, L., Eybpoosh, M., Akinci, B.: Developing As-built Building Information Model Using Construction Process History Captured by a Laser Scanner and a Camera. Construction Research Congress (2012)

18. American Institute of Architects, Integrated Project Delivery: A Guide. CA Council (2007)

19. Glick, S., Guggemos, A.: IPD and BIM: Benefits and opportunities for regulatory agencies. In: Proc. of 45th Associated Schools of Construction National Conference, Gainesville (2009)

20. Bew, M., Underwood, J.: Delivering BIM to the UK Market. In: Underwood, J., Umit, I. (eds.) Handbook of Research on Building Information Modeling and Construction Informatics: Concepts and Technologies, pp. 30-64. IGI Global (2010)

21. London, K., Singh, V., Taylor, C., Gu, N., Brankovic, L.: Building information modelling project decision support framework. In: Dainty, A. (ed.) Proc. 24th Annual ARCOM Conference, Cardiff, UK, vol. 2, pp. 655-664. Assoc. of Researchers in Const. Mgmt (2008)

22. Yin, R.K.: Case Study Research: Design and Methods, 4th edn. Sage Pub., CA (2009)

23. East, W.E.: Performance Specification for Building Information Exchange. National Institute of Building Sciences and buildingSMART Alliance (2009)

24. Penttilä, H.: Describing the changes in architectural information technology to understand design complexity and free-form architectural expression. ITcon 11 Special Issue: The Effects of CAD on Building Form and Design Quality, 395-408 (2006) 